\title{
La educación virtual durante la pandemia COVID-19: realidades y retos
}

\author{
Diana Sibaja \\ Universidad de Iberoamérica
}

\section{Resumen}

Con la pandemia del COVID-19, la educación en todos los niveles, ha atravesado cambios importantes, convirtiéndose en retos para cada docente y para cada persona estudiante. Antes de esta pandemia, se hablaba de la educación virtual y se contaban con ofertas educativas que trabajaban desde esta modalidad, no obstante, desde hace un poco más de un año, cada sistema educativo debió realizar adaptaciones de forma rápida, para continuar con procesos de enseñanza y aprendizaje por medios virtuales con las poblaciones con las que se trabaja. Un año después, se hace la reflexión sobre qué es la educación virtual, cuáles son sus características, cuál es el rol docente, el rol del estudiante y los beneficios que esta trae para lograr aprendizajes significativos y, a la vez, crear conciencia sobre las posibles necesidades que pudieran existir en la comprensión y aplicación de la educación virtual en las comunidades educativas, lo cual involucra desde las personas encargadas de tomar decisiones en cada institución educativa (administración), hasta las personas que construyen el aprendizaje, siendo necesario identificar los desafíos para realizar los ajustes necesarios involucrando la ética, el profesionalismo y la responsabilidad de lo que conlleva realmente generar aprendizaje desde la modalidad virtual.

Palabras Clave: Educación virtual, Educación Universitaria, E-learning, aprendizaje

\begin{abstract}
With the COVID-19 pandemic, education, at all levels, has undergone important changes, that became important challenges for each professor and student. Before this pandemic, some educational systems used e-learning, and there were educational offers that worked from this modality, however, for a little more than a year, each educational program had to make quick adaptations, to continue with processes of teaching and learning by virtual strategies with the populations with whom it works. A year later, reflection is made on what e-learning really is, what are its characteristics, what is the teaching role, the student role and the benefits it brings to achieve significant learning and, at the same time, this written essay wants to create awareness about the possible needs that may exist in the understanding and application of elearning in educational communities, which involves from the persons who are in charge of make important decisions (administration area), to individuals who build learning and even students, being necessary to identify the challenges to make the adjustments, that involves ethics, professionalism and the responsibility in any decision that entails to generate real learning through e-learning modality.
\end{abstract}

Keywords: Virtual Education, College Education, E-learning, learning. 


\section{Introducción}

Actualmente, por la situación de la pandemia, se han tenido que enfrentar cambios importantes en las formas tradicionales en que se han venido dando los procesos de aprendizaje, especialmente, la educación presencial, en donde se han presentado variantes significativas al tener que hacer adaptaciones con herramientas virtuales a cursos que fueron diseñados para ser presenciales, lo cual ya implica un gran reto, pero además, sin el entrenamiento suficiente para poder utilizar diferentes herramientas desde la virtualidad y generar un aprendizaje significativo.

Para comprender bien cada escenario, es indispensable aclarar los diferentes términos que últimamente se han hecho muy comunes de escuchar, empezando por "educación virtual" o "e-learning (electronic learning)" que puede definirse como "el conjunto de espacios de enseñanza -aprendizaje virtuales que se desarrollan a través de una infraestructura de redes electrónicas en internet, con la orientación de un tutor". Esto implica desde una propuesta pedagógica articulada, la forma de gestionar los procesos, el recurso tecnológico necesario, rol de las personas participantes (tanto quien da la tutoría como quien la recibe) y otros elementos necesarios para garantizar el éxito. En este punto, es indispensable señalar que deben darse tres variables importantes: un emisor que conozca las herramientas necesarias, un mensaje muy claro y sin interferencias y un receptor con las capacidades cognitivas e instrumentales para recibir el mensaje e interpretarlo de la forma correcta (Protea, 2020 parr.4)

Romero-Mayoral et al. (2014), explican que, dentro del "e-learning" hay varios modelos, los cuales, se detallan a continuación, considerándose importantes conocer desde la labor docente en entornos virtuales y así, utilizar el que mejor se ajuste, según lo qué se va a enseñar, a quiénes se va a enseñar y cómo se va a enseñar:

- B-learning (blended learning): procesos de enseñanza y aprendizaje, en los cuales se combina la modalidad presencial con la virtual.

- B-learning 3D: los estudiantes se encuentran en un entorno 3D simulado.

- MMOL (Massively Multiuser Online Learning ): entorno virtual de aprendizaje en $3 \mathrm{D}$, en donde estudiantes y docentes pueden interactuar y crear escenarios para el aprendizaje.

- M-learning: modalidad de aprendizaje en la cual se utilizan aparatos electrónicos como smartphones, agendas electrónicas, tablets y cualquier dispositivo que contenga conectividad inalámbrica.

- U-learning: (universal e-learning): adecúa contenidos en diferentes medios como video conferencias, smartTV, smartphone, entre otros, por distintas herramientas tecnológicas que faciliten los procesos de enseñanza y aprendizaje. 
- R-learning (rapid e-learning): es la elaboración de documentos sencillos con información actual sobre una temática para capacitar a un número de personas en un espacio de tiempo corto. Estos documentos suelen tener una vigencia limitada y con temáticas actuales.

- W-learning (workflow learning): aprendizaje a través de espacios cortos, por ejemplo, entrevistas a expertos, entre otros de este tipo.

Por otro lado, está el concepto de entorno virtual de aprendizaje que se refiere a un espacio de interacción que posibilita procesos de enseñanza y aprendizaje, por medio de una selección adecuada de materiales y actividades desde el aprendizaje colaborativo, utilizando plataformas tecnológicas creadas para este fin y que resultan en la motivación, interés por aprender, autocontrol, autonomía y otras variables que son propias del crecimiento personal (Protea, 2020).

Además de lo anterior, es necesario definir lo que es la educación presencial, la cual es un espacio de interacción en donde un docente imparte la clase a sus estudiantes en un mismo lugar y tiempo. (Torrealba Peraza, 2004 citado en Romero-Mayoral et al. 2014).

En la educación presencial pueden aplicarse diferentes metodologías como lo son clases magistrales, dinámicas, juegos, debates, entre otros, ya que se aprovecha lo mejor posible el estar en el mismo lugar y tiempo, siendo que, desde la comunicación, esto es una característica óptima, ya que permite, esta comunicación entre la persona docente y el estudiantado y, el profesorado puede interpretar si las personas estudiantes han comprendido o no un contenido, pudiendo utilizar otras formas para explicarlo en el momento y lograr que tengan una mejor comprensión de lo que se explica. También, la persona docente puede observar el lenguaje corporal de sus estudiantes, permitiéndole percibir las relaciones del grupo, vínculos, nivel de interés, cansancio y otros factores que pueden influir de forma positiva o negativa en el aprendizaje, lo cual le permite realizar los ajustes necesarios en el tiempo y lugar en el que la dinámica se está dando (Torrealba Peraza, 2004 citado en RomeroMayoral et al. 2014). 


\section{Proceso de enseñanza aprendizaje}

Al tener definidos los conceptos anteriores, se hace necesario conversar sobre diferentes aspectos que están relacionados con los procesos de enseñanza y aprendizaje, iniciando con las características que la persona facilitadora debe mostrar al trabajar desde la modalidad virtual:

En cuanto al perfil docente, Gargallo, Sánchez, Ros y Ferraras (2010) realizaron un estudio con 50 personas estudiantes e investigaron sobre las habilidades o cualidades de un buen profesor (a), y encontraron características como:

- Comprensivos

- Respetuoso

- Capacidad de escucha

- Responsabilidad

- Buena preparación de clases

- Buena comunicación

- Capacidad de enseñar estrategias para trabajar el contenido y aprender

- Capacidad de motivar a sus estudiantes

- Uso de ejemplos, casos prácticos y reales

- Uso de diferentes metodologías en el aula

- Escogencia de materiales que generen aprendizaje significativo (lecturas).

- Uso de evaluación formativa

Las características enumeradas anteriormente, son aspectos que deben ser parte de cualquier persona docente en ambientes educativos, independientemente de si se trabaja en modalidad presencial o virtual, para así garantizar el éxito de cualquier proceso de enseñanza y aprendizaje que se esté impartiendo.

Por otro lado, dentro del perfil docente, específicamente en entornos virtuales de aprendizaje, se menciona que, además de lo anterior, la persona docente debe mostrar conocimiento y utilización de las herramientas informáticas y telemáticas; conocimiento y utilización de técnicas de planificación y diseño en el ámbito virtual; conocimiento y utilización de metodologías didácticas para el e-learning y el b-learning, así como también, el diseño de materiales, el dominio de contenidos y la planificación del proceso de aprendizaje (Blázquez y Alonso, 2009; Imbernón, Silva y Guzmán, 2011; Sotelo Castillo et al, 2017 p. 79). 

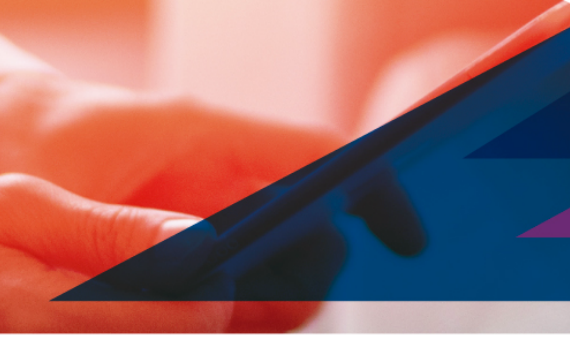

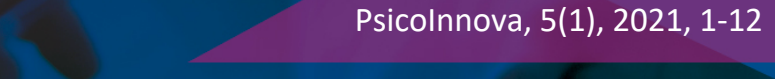

Una de las mayores dudas que genera el e-learning es sobre la temática de las emociones, lo cual juega un papel fundamental en el aprendizaje, no obstante, en entornos virtuales el aspecto emocional puede considerarse como "poco", y esto puede generar dificultades en que la persona se sienta parte de una comunidad educativa, pudiendo afectar su proceso de aprendizaje. Muchos profesionales que ejercen la docencia comentan, de forma constante, la importancia de ese contacto humano en los procesos de aprendizaje que se imparten y que, por la modalidad virtual estos parecieran no lograrse de la misma manera, ya que la dinámica en el entorno virtual es una relación entre la persona estudiante y su docente o entre las personas estudiantes cuando realizan trabajos grupales, pero al utilizar herramientas tecnológicas para lograr esto, el vínculo y las relaciones sociales pueden no crearse de la misma manera, siendo una de las causas en la disminución de ese contacto humano.

Para lograr procesos de enseñanza y aprendizaje de calidad es necesario poder brindar las herramientas y capacitaciones necesarias al equipo docente, ya que cada persona docente debe estar comprometida con el modelo de la virtualidad, evitar replicar la clase presencial en la virtualidad y garantizar la formación necesaria en el uso de plataformas tecnológicas que permitan facilitar el aprendizaje, siendo estos puntos no solo responsabilidad de la persona docente, sino que también, del área administrativa de cada institución para lograrlo de mejor manera, así como también, la importancia de capacitaciones con las debidas actualizaciones en la información.

Es importante mencionar que con las tecnologías no es que se aprende más o menos, si no que la forma de generar aprendizaje es diferente, y esto es parte de lo que debe conocerse desde el rol del facilitador para crear aprendizaje, lo cual va desde la temática que se desea enseñar, escoger los materiales y actividades de aprendizaje, hasta la evaluación y contemplar los ajustes en todo el proceso, siendo así que, una plataforma virtual por sí sola o un conocimiento significativo de herramientas tecnológicas no son sinónimo de éxito en el aprendizaje.

Otro de los puntos necesarios de contemplar es el aprendizaje colaborativo, ya que este tipo de metodología permite un aprendizaje entre compañeros y compañeras, sin jerarquías ni autoritarismos, pero sí con un diseño, organización y apoyo que genere justamente aprendizaje significativo mediante ambientes flexibles.

El trabajo colaborativo puede definirse como el conjunto de métodos de instrucción y entrenamiento que propician el desarrollo de habilidades mixtas, en donde cada persona del grupo es responsable de su propio aprendizaje y del de los demás (Lucero, 2003, citada en Rodríguez Mora, 2019). 
Al utilizar el trabajo colaborativo en entornos virtuales de aprendizaje (EVA que, permiten crear comunidades virtuales para aumentar el aprendizaje en determinados contenidos), los resultados en los procesos de enseñanza y aprendizaje son positivos, siendo este un gran reto, ya que, al utilizar la tecnología como un medio para facilitar estos procesos, una cantidad importante de docentes, insisten en utilizar la educación tradicional o estrategias tradicionales, lo cual lleva a un error y posible fracaso en lograr los objetivos de aprendizaje planteados para sus estudiantes.

El término utilizado para el aprendizaje colaborativo en entornos virtuales de aprendizaje o facilitado por medio de una computadora se conoce como Computer Supported Collaborative Learning (CSCL), implicando un espacio de interacción constante para generar el aprendizaje.

Al utilizar el trabajo colaborativo en los EVA, es necesario tener la claridad suficiente sobre los cambios que poseen tanto las personas facilitadoras, como los y las estudiantes, empezando porque la persona facilitadora no es una figura autoritaria, si no que más bien guía y facilita los procesos de enseñanza y aprendizaje. Por otro lado, se promueve la interacción de todos los miembros del grupo a través de diferentes recursos que proveen los EVA, como lo son los foros, lluvia de ideas, videoconferencias, comunicación sincrónica y asincrónica, entre otras partes. En cuanto al rol de la persona estudiante, esta debe poseer cierto grado de autonomía y ser consciente de que es responsable de su propio aprendizaje y el de los demás, por lo que es importante cada aporte que realiza desde los diferentes recursos, qué información brinda y cómo la comparte, cómo se relaciona con su grupo y el facilitador, uso de las reglas de netiqueta (reglas para trabajar y comunicarse desde los entornos virtuales de aprendizaje), entre otros aspectos que son diferentes al modelo tradicional, en donde el docente es quien está a cargo de los procesos de enseñanza y aprendizaje y la persona estudiante solamente recibe información y se le dice de forma puntual qué debe hacer y cómo debe hacerlo, de ahí que el trabajar desde la virtualidad en educación requiere un cambio de paradigma y una comprensión de lo que es y conlleva, pero sobre todo, una disposición de parte de la comunidad educativa para lograr mediaciones pedagógicas virtuales que favorezcan el aprendizaje.

Para lograr procesos de enseñanza y aprendizaje que puedan considerarse, mayoritariamente, exitosos, se hace necesario que las áreas administrativas de cada institución educativa comprendan qué conlleva y en cuáles temáticas, cursos u otros el trabajar desde entornos virtuales y el e-learning generan aprendizajes significativos. Actualmente, con la situación de la pandemia, el uso de la tecnología para crear y continuar con espacios de aprendizaje ha sido utilizado casi por la totalidad de los centros de enseñanza y es, razonable por la situación actual, no obstante, al atravesar esta situación, deben contemplarse variables básicas pero que son determinante para brindar entornos de aprendizaje de calidad y es qué se quiere aprender, 
a quiénes va dirigido, cómo se va a lograr este aprendizaje y por medio de qué, siendo necesario estudiar a profundidad estas variables para así tomar las mejores decisiones sobre cuál modalidad utilizar y no solo, reducir esta decisión a la popularidad que se ha generado en el último año, especialmente.

Otro punto determinante es que se comenta mucho sobre la afinidad del uso de la tecnología de las personas jóvenes y que este criterio pudiera ser necesario para virtualizar cualquier programa educativo. En este punto, Cedeño Romero (2019) realizó una investigación en donde se encuestó a 100 estudiantes que cursaban el bachillerato en la Unidad Educativa Ambato para describir las actitudes de los alumnos que han empleado las aulas virtuales a través de sus experiencias, específicamente conocer el criterio que tienen los estudiantes sobre la organización de las clases, las estrategias pedagógicas implementadas y definir el tipo de interacción comunicativa que se establece entre el alumnado y la persona docente durante el proceso de aprendizaje.

Por lo anterior, se discutió la importancia de un ambiente virtual de aprendizaje, en donde Rivera (2013 citado en Cedeño Romero, 2019) hace énfasis en la planificación cuidadosa basada en las características y necesidades de la población estudiantil a cargo, así como el uso de teorías educativas que generen en aprendizajes significativos. Indica, también, que no es solo una nueva forma de la educación presencial, sino que es necesario comprender que es una forma nueva de enseñanza, un cambio hacia un nuevo paradigma, para responder a las características de la nueva sociedad digital que enfrentamos, que incluye la alfabetización digital. Además, advierte que, detrás de estos ambientes, hay un gran trabajo por parte del docente, desde el diseño instruccional, hasta su implementación y, se comenta la importancia de mejorar la interacción con los estudiantes y las realimentaciones de los contenidos.

Igualmente, se indica que la tecnología es parte de los y las jóvenes, por lo que, para ellos, la educación también forma parte de esto, es decir, no solo se da en un aula y a través del docente, pero la planificación y uso adecuado del entorno virtual es lo que hará exitoso un proceso de enseñanza y aprendizaje, no solo tomar en cuenta que, al ser la tecnología parte de la cotidianeidad de las personas en general y, especialmente de los jóvenes, es que cualquier proceso educativo puede impartirse bajo esta modalidad. Además, debe contemplarse que en Costa Rica muchas zonas y personas no cuentan con las herramientas necesarias y/o no tienen conexión en sus zonas, siendo esto una desventaja en la educación y siempre debe contemplarse los espacios inclusivos en cada proceso educativo que se planee o se quiera impartir. 

Con lo anterior, al investigar lo que implica la enseñanza virtual, se requiere de forma urgente que, las áreas administrativas o jefaturas de las instituciones educativas que toman decisiones sobre modalidades de aprendizaje, tienen el gran reto de investigar y realizar las acciones necesarias para realizar todos los ajustes que requiere impartir educación desde la virtualidad, lo cual es dejar de darle énfasis a adquirir plataformas virtuales y continuar procesos tradicionales por estas vías, sino, comprometerse a que su comunidad educativa, especialmente los equipos docentes sean correctamente capacitados para que sean especialistas en enseñanza virtual y que generen formas de aprendizaje responsables, éticas y profesionales, de lo contrario, se continuará con las falsas ideas, por ejemplo, que este tipo de enseñanza es poco funcional y, además, con posibles estudiantes que deserten un curso y que podría ser por las estrategias utilizadas, más que por una dificultad real de la enseñanza virtual, de ahí la necesidad real de trascender del uso tradicional utilizando plataformas como Teams, Zoom, G-Suite, entre otras, para promover la calidad educativa en cada proceso que se tenga a cargo.

Otro punto necesario que las administraciones de las instituciones educativas deben contemplar es la anuencia y posibilidades para invertir en aspectos, como por ejemplo, que el facilitador brinde un acompañamiento diario, el soporte que conlleva la plataforma o medios tecnológicos que se decidan utilizar, personal de equipos técnicos disponibles ante cualquier eventualidad en cualquier momento del día, capacitación adecuada a las personas facilitadoras, esto de forma constante y significativa, entre otras áreas que no pueden dejarse de lado para garantizar calidad educativa y ofrecer a los estudiantes diferentes formas innovadoras para construir su aprendizaje.

En la educación superior o universitaria, es necesario contemplar las variables de cada carrera y cada conocimiento, habilidad y competencia por desarrollar para así, decidir si la modalidad que mejor prepara al estudiante sería presencial, virtual o bimodal, siendo una decisión también que debe contemplar la calidad educativa y desde cuál modalidad, trabajar. Por ejemplo, en la carrera de Psicología, se hace necesario poder aplicar y revisar pruebas psicológicas para diferentes poblaciones y en diferentes áreas, por lo que los cursos que generan aprendizaje sobre esto, idealmente, se requiere la presencialidad y, bajo la guía del docente, poder aplicar las diferentes pruebas, resaltando las fortalezas, corrigiendo los errores y así acercar al estudiante a contextos reales del rol del profesional en psicología. También pueden nombrarse muchos ejemplos más en diferentes carreras profesionales, insistiendo que las decisiones sobre la modalidad que puede generar de mejor forma procesos de aprendizaje, deben valorarse a la luz de lo que mejor resulta en términos de aprendizaje. 
Por último, está el rol del estudiante, en donde también, este rol desde lo virtual, tiene características necesarias de mencionar y que también son parte del éxito de la educación virtual.

Rizo Rodríguez (2020) define características como la capacidad de autogestión, responsabilidad, autodisciplina, autoaprendizaje, análisis crítico, reflexivo y el trabajo colaborativo son necesarias para que el resultado sea el esperado, además de ser consciente de su interacción y aporte de forma ética y responsable con él mismo y con su grupo de compañeros para llegar al nivel de aprendizaje óptimo.

Rizo Rodríguez (2020) menciona diferentes roles del estudiante desde los entornos virtuales:

- Orientado al fortalecimiento de la autodisciplina: habilidades para cumplir metas que son deseables, en donde se involucra gestión del aprendizaje, dedicación en las tareas a cargo que tiene que ver con la fundamentación de objetivos, su ejecución y seguimiento, además de la motivación, decisión y perseverancia para lograr las metas.

- orientado al mejoramiento del autoaprendizaje: capacidad de aprender de forma autónoma, activa y participativa, obteniendo conocimiento, habilidades y valores que generen la autoformación de la persona. Lleva al estudiante a ser autodidacta y autoreflexivo.

- Orientado al fortalecimiento del análisis crítico y reflexivo: habilidad para razonar, analizar y argumentar posturas, hechos, conocimiento y otros. Busca que los estudiantes sean sujetos pensantes, actuantes, creadores y constructores de saberes individuales y sociales.

- Orientado al mejoramiento del trabajo colaborativo: cualidades individuales que son compartidas entre pares y facilitadores a través de herramientas como el foro, el correo electrónico, el chat y otros. Además, se preocupa por su propio aprendizaje y el del grupo de estudiantes.

Con lo anterior, desde la experiencia docente, es necesario que los sistemas educativos escolares promuevan el desarrollo de estas habilidades, contemplando también el apoyo por parte de los padres y madres de familia para lograr que estas habilidades vayan desarrollándose lo mejor posible, en donde, se hace necesario, nuevamente, resaltar la importancia que los docentes apliquen formas innovadoras de generar aprendizaje por medio de estas habilidades, siendo necesario salir de las formas tradicionales, en donde el estudiante, desde el rol pasivo, difícilmente logrará un nivel óptimo en estas capacidades, por lo que, al requerirlas en la educación virtual, esto podría influir en un proceso difícil de adaptación para el estudiante y, en otros casos, terminar desertando el programa educativo 
en el que esté porque podría sentirse desubicado, confuso e incapaz de hacerse cargo de su propio proceso y del de los demás miembros del grupo, por lo que, también, el autoaprendizaje y la autodisciplina deben promoverse desde edades tempranas, no solo en ambientes escolares, si no en la cotidianeidad del niño o niña y así irlas fortaleciendo, siendo necesarias para aprender de forma significativa y no solo aprobar niveles educativos, lo cual son discusiones y recomendaciones que han estado en la literatura desde hace tiempo atrás, pero todavía hay grandes dificultades para ponerlas en práctica, crear entornos para facilitar estas capacidades se ha vuelto un reto importante en nuestra educación costarricense en los diferentes niveles, por lo que debe continuarse trabajando por hacer la conciencia y capacitar de forma real a los responsables de cada procesos de enseñanza y aprendizaje. Actualmente, muchos estudiantes todavía esperan que el docente sea quien le dice qué hacer y cómo hacerlo y, les resultad difícil crear por sí solos y adueñarse de su propio proceso de aprendizaje y es ahí en donde debe reflexionarse sobre el rol docente y el rol del estudiante.

Como retos finales, se concluye la importancia de comprender, analizar y aplicar en cada programa educativo las diferentes estrategias que lleven a crear de forma significativa el aprendizaje deseado y necesario, siendo importante retomar las preguntas básicas en la educación: qué se quiere enseñar, a quién se quiere enseñar, cómo se quiere enseñar y por medio de qué se enseñará, para desde ahí tomar las decisiones que resulten en calidad educativa, siendo ejes transversales la responsabilidad, la ética, la capacitación real y el profesionalismo para decidir y realizar los ajustes necesarios para innovar y crear espacios reales de aprendizaje que contribuirán a la sociedad en general y especialmente, al desarrollo de nuestro país.

\section{Referencias}

Blázquez, F., y Alonso, L. (2009). Funciones del profesor de e- learning. Revista de Medios $y$ Educación, 34(34), 205-215.

Cedeño Romero, E.(2019). Entornos Virtuales de Aprendizaje y su Rol Innovador en el Proceso de Enseñanza. Revista de Ciencias Humanísticas y Sociales 4(1) pp. 119127. DOI: https://doi.org/10.5377/multiensayos.v6i12.10117

Gargallo, B., Sánchez, F., Ros, C. y Ferreras, A. Estilos docentes de los profesores universitarios: La percepción de los alumnos de los buenos profesores. Revista Iberoamericana de Educación, 51(4), 1-16. DOI: https://doi.org/10.35362/RIE5141826. 


\section{U pesco}

Imbernón, F., Silva, P., y Guzmán, C. (2011). Competencias en los procesos de enseñanzaaprendizaje virtual y semipresencia. Comunicar, XVIII(36), 107-114. DOI: https://doi.org/10.3916/C36-2011-03-01

Protea (2020). El reto docente en un contexto social en crisis, oportunidades desde la virtualidad. $\quad$ https://www.ucr.ac.cr/noticias/2020/04/01/el-reto-docente-en-uncontexto-social-en-crisis-oportunidades-desde-la-virtualidad.html

Rizo Rodríguez, M.(2020). Rol del Docente y del Estudiante en la Educación virtual. Revista $\begin{array}{llll}\text { Multi-Ensayos, } & 6(12), & 28-37 . & \text { DOI: }\end{array}$ https://doi.org/10.5377/multiensayos.v6i12.10117

Rodríguez Mora, Y (2019). Aprendizaje colaborativo en entornos virtuales [Tesis de maestría, Universidad Andina Simón Bolívar]. Repositorio UASB. https://repositorio.uasb.edu.ec/bitstream/10644/7217/1/T3113-MINE-RodriguezAprendizaje.pdf

Romero-Mayoral J; García-Domínguez M; Roca-González, C; Sanjuán, Pérez, A; PulidoAlonso, A.(2014). Diseño de un aprendizaje adaptado a las necesidades del alumno. Teoría de la Educación. Educación y Cultura en la Sociedad de la Información, 15(3), 172-189

Sotelo Castillo M; Vales García, J; García López, R; Barrera Hernández, L. (2017). Características del Buen Profesor De Modalidad Presencial Y Virtual Desde La Perspectiva De Los Estudiantes. European Scientific Journal, 13(13), 78-89. DOI: http://dx.doi.org/10.19044/esj.2017.v13n13p78 